\title{
EDUCATION
}

\section{Promoting Patient Safety Through Interprofessional Education Simulation}

\section{KATIE CAVNAR, JILL VAN DER LIKE, LELA HOBBY-BURNS}

\begin{abstract}
An interprofessional education simulation project was created for Clinical Laboratory Sciences students to promote patient safety skills. Hand hygiene and patient identification were addressed in the scenario. CLS students participated in two IPE SIM experiences spaced six weeks apart. All students were educated by nursing faculty on careful hand hygiene $(\mathrm{HH})$ when entering and exiting a patient's environment. Students were separated into two groups. The control group had no further education. The intervention group were instructed on the WHO 6-step $\mathrm{HH}$ process, and rehearsed on the steps. Students took pre and post-simulation quizzes on knowledge of $\mathrm{HH}$. There was no significant difference in the quiz test scores between the two groups. Students' actual $\mathrm{HH}$ was video recorded prior to entering the patient environment and again as they exited. The intervention group demonstrated a significant and sustained increase in pre-patient $\mathrm{HH}$ times compared to the control group.
\end{abstract}

ABBREVIATIONS: IPE SIM- Interprofessional Education Simulation, HH- Hand Hygiene, WHOWorld Health Organization, ID- Identification. HAIHealthcare Acquired Infections, CLS-Clinical Laboratory Sciences

INDEX TERMS: Interprofessional Relations, Hand Hygiene, Simulation Training, Patient Identification Systems

Clin Lab Sci 2017;30(4):228

Katie Cavnar, MS, MLS (ASCP), University of West Florida. College of Health, Pensacola, FL

Jill Van Der Like, DNP, MSN, RNC, University of West Florida, College of Health, Pensacola, FL

Lela Hobby-Burns, DNP, MSN APHN-BC, University of West Florida, College of Health, Pensacola, FL

\author{
Address for Correspondence: Katie Cavnar, University of \\ West Florida, Bldg. 58, Room 76B, 11000 University \\ Parkway, Pensacola, FL 32514, 850-474-2887, \\ kcavnar@uwf.edu
}

\section{INTRODUCTION}

All healthcare students must become proficient with two critical patient safety practices: careful patient identification and hand hygiene. Wrongly identified patients or mislabeled specimen identification are sources of medical errors that jeopardize patient safety. The number one national patient safety goal of the Joint Commission (01.01.01) emphasizes improved patient identification (ID) practices. $^{1}$ Simulated patient scenarios (SIM) provide students the opportunity to practice communication skills with "patients", including introducing themselves and requesting and verifying patient ID information. Deliberate practice reinforces careful patient identification, so the correct treatment is given to the right patient, every time. A patient simulation environment offers a safe, realistic practice setting for students to gain these skills. ${ }^{2}$

Hand Hygiene $(\mathrm{HH})$ is a critical habit that must be learned and practiced before a student graduates and becomes a healthcare worker. Healthcare acquired infections (HAI) affect hundreds of millions of people worldwide. In the United States alone, estimates are that 99,000 deaths are caused by healthcare acquired infections (HAIs) every year. ${ }^{3}$ The cost of treatment for these infections can range annually from $\$ 28.4$ to 45 billion. ${ }^{3}$ Studies find that $\mathrm{HH}$ is critical for prevention of the transmission of HAIs and saving lives. ${ }^{4}$ However, this seemingly simple task is problematic, with reported $\mathrm{HH}$ compliance rates from $34-50 \%$ for healthcare workers. ${ }^{3,4}$ A recent study has shown evidence that the World Health Organization's (WHO) 6-step process $\mathrm{HH}$ technique significantly reduces bacterial count more than the basic $\mathrm{HH}$ technique. ${ }^{5}$

Patient identification and handwashing are emphasized 
to Clinical Laboratory Science (CLS) students in the classroom. However, little is known about CLS students' performance of these basic patient safety skills when they are in the workplace. There have been no published studies to our knowledge on CLS students' skills on patient ID and HH. The SIM environment is an ideal place for this introduction to best patient safety practices. This research study describes the results of 2 SIM experiences involving deliberate practice in patient safety.

\section{MATERIALS AND METHODS}

This was an interprofessional education (IPE) project between the CLS and Nursing Departments faculty and students. The simulations took place in the Nursing Skills and Simulation Learning Center (NSSL). During the SIMs the CLS students were monitored for $\mathrm{HH}$ and patient identification practices. All CLS students were juniors in their first year of the CLS program, and did not have clinical experience.

There were 2 IPE SIM experiences spaced six weeks apart. CLS students were assigned to either the intervention $(\mathrm{N}=15)$ or control group $(\mathrm{N}=12)$. Both groups were oriented by nurse faculty to the IPE SIM scenario during a pre-SIM conference. Nurse faculty reinforced key safety practices of $\mathrm{HH}$ upon entering and exiting the patient environment, and patient identification.

Additionally, on the SIM day one only, nurse faculty taught the intervention group the World Health Organization 6-step $\mathrm{HH}$ process. This is the same $\mathrm{HH}$ best practice regularly taught to nursing students. The WHO 6-step process involves more manipulation of hand washing than basic $\mathrm{HH}$, including specifically scrubbing palms several different ways, interlacing fingers, specifically rubbing backs of hands and fingers, and rotational rubbing of thumbs. ${ }^{6}$ The students practiced the 6-step process with the nursing faculty. On SIM day one only, the control group was reminded by the nurse faculty to practice proper $\mathrm{HH}$ carefully, but did not demonstrate or rehearse it.

During the IPE SIM experience, the CLS students were to practice these skills:

- Perform HH when entering the patient's environment,

- Introduce themselves, explaining the purpose of their visit to withdraw blood specimen,
- Conduct careful patient identification by verbal verification, and by comparing the patient ID armband for match with specimen request information;

- Simulate blood specimen withdrawal at the patient bedside,

- Safely leave the patient environment by again performing $\mathrm{HH}$.

In the first SIM, CLS students practiced entering the patient's environment to obtain a blood specimen and the CLS students themselves acted in roles as the patient and patient's partner; in the second SIM, there were complications with the patient, and nursing students were involved with the patient at the bedside. The patient in the second SIM scenario was a Laerdal maniken that the nursing students performed interventions on while the CLS students collected blood samples. The nursing students were treating the patient for Disseminated Intravascular Coagulation (DIC) of unknown origin while the CLS students were entering the area, working with and around nursing students to obtain blood samples, and still practicing $\mathrm{HH}$ and patient identification.

The IPE SIM scenarios focused on three outcomes to measure patient safety knowledge and skills:

1. Cognitive knowledge about hand hygiene was determined by a pre-quiz before the first SIM clinical day and, a post-quiz after the last SIM clinical day.

2. Demonstration of patient identification before beginning blood specimen procedure.

3. Hand-washing time was measured in seconds at the sink, when entering and exiting the patient environment. Image 1 shows a student performing $\mathrm{HH}$ during the SIM. (Figure 1)

To verify the psychomotor skills of the CLS students an overhead video camera recorded all $\mathrm{HH}$ activities at the wash basin. A second overhead video camera recorded the patient's environment. For both IPE SIM scenarios, an observer watched the video recordings in a remote location noting the time duration the CLS students performed $\mathrm{HH}$ when entering and exiting patient environment, and the students' patient identification verification skills. Students were aware that they were being recorded, but they were not aware that their handwashing was being evaluated. The control group was taught the WHO 6-step HH process at the end of 
the second SIM and given practice time to improve their HH skills.

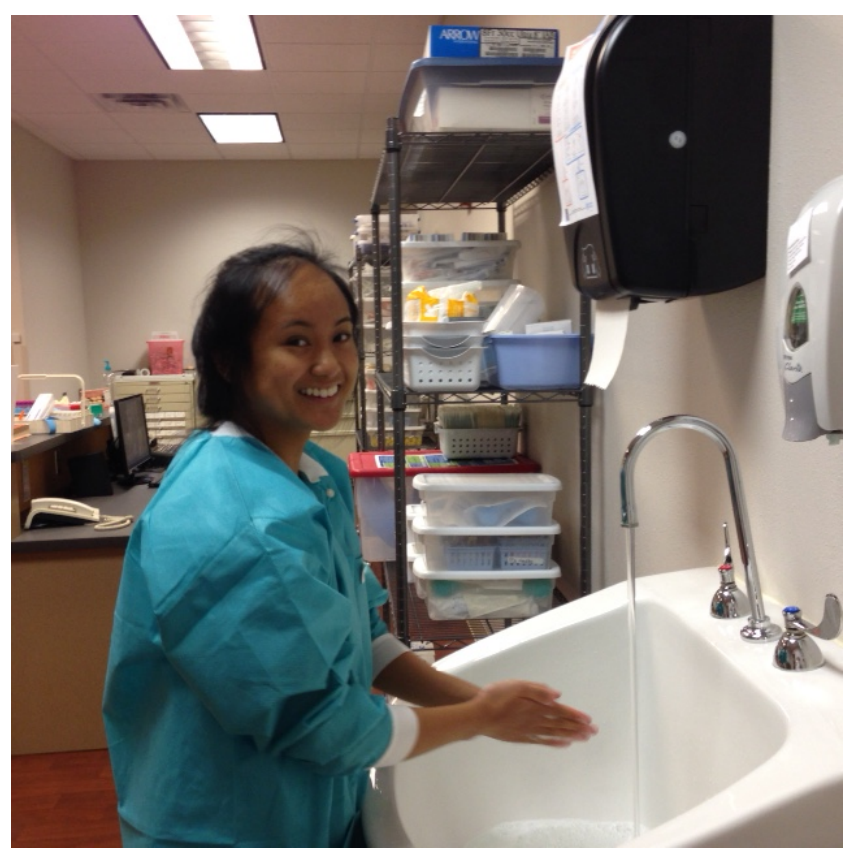

Figure 1. CLS student performs handwashing as per WHO 6-step guidelines during simulated lab experience.

Institutional Review Board (IRB) approval was attained from the University of West Florida. All students signed an informed consent. Statistical analysis was performed with SPSS (IBM).

\section{RESULTS}

A paired samples $t$ test found no significant difference between the intervention and control groups for the pre and post knowledge quiz. Pre and post written test scores were compared for the intervention group and the control group using a 2-tailed Students $t$ test. There was no difference between the pre-scores $(\mathrm{p}=0.588)$ or the post-scores $(p=0.7560)$ for both groups. Except for one student in the intervention group on SIM day one, all students accurately identified the patient with verbal verification and by comparing armband information to laboratory requisition and labels during both SIMs.

Independent samples $t$ tests were performed to assess whether mean $\mathrm{HH}$ time differed significantly for the intervention group participants who received the WHO 6-step $\mathrm{HH}$ deliberate practice instruction, compared with the 12 control group participants who did not receive the training. The time of hand washing in seconds was analyzed for normality. Based on Kolmogorov-
Smirnov and Shapiro-Wilk test, both pre-patient and post-patient hand washing times showed nonsignificance, indicating normality.

SIM Day one Hand Hygiene $(\mathrm{HH})$ mean times were compared before entering (pre) patient's environment. The intervention group washed their hands for a statistically significant longer period of time before patient contact than the control group: Intervention group $\mathrm{HH}$ time 48.7 seconds, control group 33.0 seconds $(\mathrm{p}<0.002)$. There was no statistically significant difference between the two groups when leaving the patient environment despite a 9-second difference $(\mathrm{p}=$ 0.119). See Table 1.

On SIM Day two, 6-weeks later, the intervention group again performed longer $\mathrm{HH}$ time pre-patient environment, 50 seconds, compared to control group's 37 seconds $(\mathrm{p}<0.026)$. The post-patient environment $\mathrm{HH}$ time comparison found no statistical $\mathrm{HH}$ time difference between the groups.

Table 1. Mean hand hygiene times on SIM Day 1 and SIM Day 2 (6-weeks later)

\begin{tabular}{cccc}
\hline Sim Day & $\begin{array}{c}\text { Intervention group } \\
\text { Mean HH time in } \\
\text { seconds (SD) } \\
\mathrm{N}=15\end{array}$ & $\begin{array}{c}\text { Control group } \\
\text { Mean HH time } \\
\text { in seconds (SD) } \\
\mathrm{N}=12\end{array}$ & (p-value) \\
\hline $\begin{array}{c}\text { Sim 1 } \\
\text { pre-patient }\end{array}$ & $48.7(10.7)$ & $33.0(12.9)$ & 0.002 \\
$\begin{array}{c}\text { Sim 1 } \\
\text { post-patient }\end{array}$ & $35.3(17.3)$ & $25.9(11.3)$ & 0.119 \\
$\begin{array}{c}\text { Sim 2 } \\
\text { pre-patient }\end{array}$ & $50.5(8.13)$ & $37.0(14.34)$ & 0.026 \\
Sim 2 & & & \\
post-patient & $39.6(8.69)$ & $34.1(12.25)$ & 0.224 \\
\hline
\end{tabular}

Table 2. Mean hand hygiene times of all students during both SIM days.

\begin{tabular}{lcccc}
\hline All students & $\begin{array}{c}\text { Pre- } \\
\text { patient } \\
\text { mean } \\
\text { (both groups) }\end{array}$ & $\begin{array}{c}\text { Post- } \\
\text { patient } \\
\text { seconds } \\
\text { mean } \\
\text { seconds } \\
(\mathrm{SD})\end{array}$ & $\begin{array}{c}\text { Paired t- } \\
\text { test }\end{array}$ & P-value \\
\hline $\begin{array}{l}\text { Both days } \\
\text { combined }\end{array}$ & $\begin{array}{c}\text { 88.2 } \\
(22.54)\end{array}$ & $\begin{array}{c}70.6 \\
(23.8)\end{array}$ & 4.67 & $<0.0001$ \\
\hline
\end{tabular}


Time spent performing $\mathrm{HH}$ pre- and post-patient environment was compared for all students during both SIM days. In the pre-patient environment, all students spent about 18 seconds longer performing $\mathrm{HH}$ than when leaving the (post) patient environment. (See Table 2).

\section{DISCUSSION}

The 2013 National Quality Strategy to reduce HAIs has drawn increased attention to $\mathrm{HH}$ practices. ${ }^{7,8}$ Implementation of the WHO recommendations for $\mathrm{HH}$ in healthcare workers has been successful with regards to compliance, especially in nurses. ${ }^{9}$ Minimal published information is found on $\mathrm{HH}$ practice and instruction of CLS healthcare workers and CLS students' education. Most of the participating CLS students had never entered a patient hospital room, much less introduced themselves to a patient or interacted with a nurse. These 2 IPE SIM scenarios provided deliberate practice for CLS students within a safe learning lab. It also provided them with basic interprofessional communication experience with other healthcare students.

Except for 1 student in the first SIM scenario, all students accurately identified the patient with two identifiers in both SIMs. This indicates faculty instruction and SIM skills development were successful.

CLS students were cognitive of $\mathrm{HH}$ practices from classroom instruction. Both intervention and control groups scored well on both the pre- and post- quizzes. However, the group that had deliberate practice performed more robust handwashing. Furthermore, the habit persisted for at least 6 weeks. Interestingly, and a point for future IPE instruction, all students, in both groups, performed HH 10.6 seconds longer when entering the patient environment than after exiting the patient environment. After the experiment was completed, the control group was instructed on the WHO 6-step $\mathrm{HH}$ process with deliberate practice and rehearsal.

This research, in addition to another interprofessional education experiences that our faculty created, ${ }^{10,11}$ demonstrates the value of augmenting classroom instruction with an IPE SIM scenario and deliberate practice of psychomotor skills. In this particular IPE SIM exercise, most of the instruction was done by nursing faculty. The CLS faculty also provide guest lectures to nursing students on various subjects including molecular testing and immunohematology. This particular IPE SIM also preceded a third IPE SIM in which the nursing students were instructed in molecular testing by CLS instructors and participated in such testing in the CLS facilities, please see Behan, KJ, "Pride and prejudice and learning: an interprofessional experience with CLS and Nursing students." ${ }^{\prime 1}$ These results add to the limited body of literature about IPE within our profession.

There are some limitations to this study. There were a small number of participants. We only measured CLS students' patient safety performance of patient identification and hand hygiene times when entering and exiting the patient environment. Future study is needed on students' reduced $\mathrm{HH}$ time after exiting patient environment, compared to entering.

Healthcare institutions struggle for staff compliance on hand hygiene for infection control and patient identification. Our research suggests that CLS students who practice their patient safety skills in an IPE SIM integrate the value of this activity into their affective behavior.

\section{ACKNOWLEDGEMENTS}

This project was funded by the University of West Florida College of Health. Carla Thompson performed the statistical analysis.

\section{REFERENCES}

1. Joint Commission 2016 National Patient Safety Goals: 2016 National Patient Safety Goals Presentation. Available from https://www.jointcommission.org/npsg_presentation/. Accessed 2016 Oct. 1.

2. McGahie WC, Issenberg SB, Petrusa ER, Scalese RJ. A critical review of simulation-based medical education research: 20032009. Med Educ. 2010;44(1):50-63.

3. Joint Commission Center for Transforming Healthcare: Bringing the leading Healthcare Organizations together to solve challenging Health Care problems. Available from http://www.centerfortransforminghealthcare.org/assets/4/6/han d_hygiene_storyboard.pdf. Accessed 2016 Oct. 1.

4. Birnbach DJ, Nevo I, Barnes S, Fitzpatrick M, Rosen, LF, Everett-Thomas R, et al. Do hospital visitors wash their hands? Assessing the use of alcohol-based hand sanitizer in a hospital lobby. Am J Infect Control. 2012;40(4):3.

5. Reilly JS, Price L, Lang S, Robertson C, Cheater F, Skinner K, Chow A. A pragmatic randomized controlled trial of 6-step vs 3step hand hygiene technique in acute hospital care in the United Kingdom. Infect Control Hosp Epidemiol 2016;37(6);661-6.

6. World Health Organization's Hand Hygiene: Why, how \& when brochure: How to handwash. Available from 


\section{EDUCATION}

http://www.who.int/gpsc/5may/Hand_Hygiene_Why_How_a nd_When_Brochure.pdf?ua=1. Accessed 2016 Sept 21.

7. Decker AS, Cipriano GC, Tsouri G, Lavigne JE. Monitoring pharmacy student adherence to World Health Organization hand hygiene indications using radio frequency identification. Am J Pharm Educ. 2016;80(3):51.

8. 2013 Annual Progress Report to Congress: National strategy for quality improvement in health care: Table 1: Priority 1 measures. Available from http://www.ahrq.gov/workingfor quality/reports/annual-reports/nqs2013annlrpt.htm.

Accessed 2016 Sept. 21.
9. Allegranzi B, Gayet-Ageron A, Damani N, Bengaly L, McLaws ML, Moro ML, et al. Global implementation of WHO's multimodal strategy for improvement of hand hygiene: a quasiexperimental study. Lance Infec Dis 2013;13(10);843-51.

10. Van Der Like J, Behan KJ. Introducing Interprofessional Education to BSN and CLS students using a simulated healthcare setting. Clin Lab Sci in press.

11. Behan KJ, Coffey K, Promo M, Brooks T, Van Der Like J. Pride and prejudice and learning: an interprofessional experience with CLS and Nursing students. Clin Lab Sci in press. 